\title{
The powder alloys of Fe-Cr-Co system heat-treated in the «ridge» region
}

\author{
K. N. Generalova ${ }^{\dagger}$ I. V. Ryaposov, A. A. Shatsov \\ †kngeneralova@mail.ru
}

Perm National Research Polytechnic University, 29 Komsomolskii pr., 614990, Perm, Russia

The hysteresis Fe-Cr-Co alloys based on the combination of producibility and mechanical and physical properties, are promising magnetic-hard materials. The powder metallurgy provides great technological advantages for designing parts from $\mathrm{Fe}-\mathrm{Cr}$-Co system. The higher hysteresis properties were obtained due to the required morphology of $\alpha_{1}$ ordered magnetic phase. Ordering of structure made it possible to produce $\alpha 1$ phase at the stage of preliminary heat treatment and further development of thermal treatment. The $\mathrm{Fe}-22,5 \% \mathrm{Cr}-15 \% \mathrm{Co}-(4 \% \mathrm{Mo})$ nanostructured powder alloys were investigated. The powders of extremely pure elements were used as their components. The use of ferrosilicon provided sintering in the presence of a liquid phase formed by contact melting, which resulting in porosity of approximately $2 \%$. The structure of the sintered samples had $\alpha$-phase with an average grain size of $90 \mu \mathrm{m}$ for samples without molybdenum and of $150 \mu \mathrm{m}$ for those with molybdenum. The subsequent tempering at $520-550^{\circ} \mathrm{C}$ caused ordering of the structure, which confirmed the fragmentation of the grain to $50 \mu \mathrm{m}$. Preliminary ordering made it possible to increase the magnetic properties twice. The required hysteresis properties were obtained after stepwise tempering in the range of $640-580^{\circ} \mathrm{C}$. After the treatments, the two-phase structure was formed. The lattice parameters of magnetic phases were determined. The powder hysteresis alloys developed, the technologies of their production and their treatment resulted in the superiority of the powder hysteresis alloys over widely used Vicalloys. These Fe-Cr-Co alloys are believed to be in the designing of hysteresis motor rotors.

Keywords: Fe-Cr-Co alloy, hysteresis properties, coercitive force, residual induction, ordering, heat treatment.

\section{Порошковые сплавы системы Fe-Cr-Co, термообработанные в области «гребня»}

\author{
Генералова К.Н. ${ }^{\dagger}$, Ряпосов И. В., Шацов А. А. \\ Пермский национальный исследовательский политехнический университет, \\ Комсомольский пр. 29, 614990, Пермь, Россия
}

Порошковые сплавы на основе системы Fe-Cr-Co (ХК) по сочетанию технологичности, механических и физических свойств являются перспективными магнитотвердыми материалами. Большие технологические преимущества получения деталей из материалов данной системы способны обеспечить технологии порошковой металлургии. Для реализации высоких гистерезисных свойств требуются заданные количество и морфология упорядоченной магнитной фазы $\alpha_{1}$. В работе, морфологию фаз и структурный состав сплавов регулировали упорядочением на стадии предварительного термического воздействия и последующей термической обработки. Исследованы наноструктурированные магнитные порошковые материалы системы $\mathrm{Fe}-22,5 \% \mathrm{Cr}-15 \% \mathrm{Co}-1 \% \mathrm{Si}-(4 \% \mathrm{Mo})$. В качестве компонентов использовали порошки особо чистых элементов. Использование феррокремния обеспечивало спекание в присутствии жидкой фазы, образующейся за счет контактного плавления. В результате спекания формировалась структура пористостью примерно 2\%. Структура спеченных образцов - альфа-фаза со средним размером зерна у образцов без молибдена - 90 мкм и 150 мкм с молибденом. Проведение последующего отпуска при температурах 520 - $550^{\circ} \mathrm{C}$ обеспечивало упорядочение структуры, что косвенно подтверждало фрагментирование зеренной структуры до 50 мкм. Предварительное упорядочение позволило повысить показатели магнитных свойств до 2 раз. Требуемые гистерезисные свойства получены после ступенчатого отпуска в интервале температур $640-580^{\circ} \mathrm{C}$. Показано, что в результате обработок формируется структура, содержащая две фазы. Определены параметры решеток магнитных фаз в порошковых сплавах. Разработанные порошковые сплавы, технологии их получения и термической обработки обеспечивают превосходство по свойствам над широко применяемыми Викаллоями. Исследованные сплавы системы Fe-Cr-Со перспективны для изготовления роторов гистерезисных двигателей.

Ключевые слова: сплав железо-хром-кобальт, гистерезисные свойства, коэрцитивная сила, остаточная индукция, упорядочение, термообработка. 


\section{1. Введение}

Создание конкурентоспособных наноструктурированных магнитных порошковых материалов с повышенными эксплуатационными характеристиками без применения экстремальных воздействий позволит существенно повлиять на прогресс в приборостроении. Две основные проблемы порошковой металлургии обусловлены неоднородностью плотности и распределения легирующих элементов. Добиться плотности и концентрационной однородности сопоставимых с лучшими традиционными материалами возможно спеканием в присутствии «исчезающей» жидкой фазы, образующейся за счет контактного плавления $[1,2]$.

Область расслоения альфа-твердого раствора на слабомагнитную $\left(\alpha_{2}\right)$ и сильномагнитную $\left(\alpha_{1}\right)$ фазы в системе железо-хром-кобальт имеет ассиметричный характер. Зависимости Точка Кюри $\left(T_{c}\right) /$ концентрация $(C)$ в широких интервалах варьирования содержания кобальта и хрома в значительной степени сдвинута в сторону меньшего содержания хрома с явно выраженными локальными максимами. Наибольшее значение функции $T_{c}=f(C)$ приходится на сплавы, содержащие $22,5 \% \mathrm{Cr}$ и $15 \%$ Со. Такие сплавы и принято называть «гребневымИ».

Гребневые сплавы обеспечивают наибольшие значения индукции и магнитной энергии. Перспективное направление применения гребневых сплавов - функционирование в слабых магнитных полях, что открывает возможность применения ХК в гистерезисных двигателях (ГД).

По данным [3, 4], требуемый уровень магнитных характеристик (коэрцитивная сила $H_{c}$, остаточная индукция $B_{r}$, максимальная напряженность поля $H_{m}$ и максимальная индукция $B_{m}$ ) могут обеспечить сплавы системы $\mathrm{Fe}-\mathrm{Cr}-\mathrm{Co}$. Одним из наиболее эффективных методов получения гребневых магнитов является порошковая металлургия, однако свойства порошковых материалов обычно существенно ниже, чем у деформированных. Другим препятствием для промышленного применения концентрационно-неоднородных сплавов является сложность реализации термомагнитной обработки (ТМО). Из-за отсутствия серийного оборудования для ТМО с высокой точностью регулирования и контроля температуры и напряженности магнитного поля.

В сплавах ХК высококоэрцитивное состояние формируется в процессе спинодального распада исходного высокотемпературного ОЦК $\alpha$-твердого раствора на две фазы: сильномагнитную $\alpha_{1}$, обогащенную железом и кобальтом, и слабомагнитную $\alpha_{2}$, обогащенную хромом.

Процесс формирования высококоэрцитивного состояния в сплавах ХК протекает в температурном интервале $700-500^{\circ} \mathrm{C}$, который условно можно разделить на два: первый $700-620^{\circ} \mathrm{C}$, в котором формируется морфология микроструктуры, второй $620-500^{\circ} \mathrm{C}$, в котором происходит окончательное концентрационное разделение фаз, их формы и размеров [5].

Цель настоящей работы - исследование структуры и свойств поликомпонентных гребневых сплавов системы ХК, полученных методами порошковой металлургии.

\section{2. Материалы и методики}

В качестве исходных компонентов шихты использовали порошки: железа ОСЧ 6-2, ТУ 6-09-05808008-262-92, хрома ПХ-1С, ТУ 14-5-298-99, кобальта ПК-1, ГОСТ 9721 - 79, феррокремния ФС50, ГОСТ1415-93, молибдена МПЧ, ТУ18-19-10.6-83. Шихту получали смешиванием компонентов в течение 8 ч в смесителе со смещенной осью вращения. Заготовки прессовали в закрытых стальных пресс-формах при давлении 600 МПА. Прессовки спекали при температуре $1350^{\circ} \mathrm{C}$ в вакууме $10^{-2}$ Па в течение 2 часов. В спеченном состоянии пористость образцов была не выше $2 \%$.

В работе использовали образцы гребневых сплавов $22,5 \mathrm{X} 15 \mathrm{KC}(22,5 \% \mathrm{Cr}+15 \% \mathrm{Co}+1 \% \mathrm{Si})$ и $22,5 \mathrm{X} 15 \mathrm{KC} 4 \mathrm{M}$ $(22,5 \% \mathrm{Cr}+15 \% \mathrm{Co}+1 \% \mathrm{Si}+4 \% \mathrm{Mo})$.

Термическая обработка гребневых сплавов включала закалку, предварительное старение и последующий отпуск. Предварительное старение заготовок проводили при $530^{\circ} \mathrm{C}$ в течение 2 часов. Первый отпуск в интервале $620-640^{\circ} \mathrm{C}$, в течение 30 мин с последующим охлаждением в воде, второй - в интервале $580-600^{\circ} \mathrm{C}$ с охлаждением на воздухе или в воде. Экспериментально установлено, что указанным интервалам температур соответствуют структурные изменения, способствующие формированию заданных значений индукции (верхний интервал температур) и коэрцитивной силы (нижний интервал температур).

Металлографический анализ проводили на микроскопе OLYMPUSGX51. Рентгенофазовый анализ проводили надифрактометре ДРОН 3 в железном $\mathrm{K}_{\alpha}$-излучении.

Магнитные свойства гребневых сплавов измеряли на микровеберметре Ф-191 в диапазоне полей \pm 90 А/см. Для этого использовали методику снятия гистерезисных свойств с цилиндрических образцов магнитотвердых материалов в слабых рабочих полях (от минус 90 до плюс 90 кА/м).

\section{3. Результаты и обсуждения}

Структура образцов из гребневых сплавов представляет $\alpha$-фазу, со средним размером зерна 90 мкм для сплава $22,5 \mathrm{X} 15$ КС и 150 мкм для сплава 22,5Х15КСМ.

Влияние низкотемпературного старения при $530^{\circ} \mathrm{C}$ на формирование зеренной структуры проявилось в фрагментации зерна до размеров 30-50 мкм (рис. 1), изменение зеренной структуры косвенно указывает на начальную стадию упорядочения [6,7]. Последующий многоступенчатый отпуск приводил к распаду высокотемпературного $\alpha$-твердого раствора на две фазы: сильномагнитную $\alpha_{1}$, обогащённую кобальтом, и слабомагнитную $\alpha_{2}$, обогащенную хромом. Температуру старения выбирали опытным путем, опираясь на измеренные значения магнитных свойств образца (таблица 1).

Для повышения коэрцитивной силы исходную шихту легировали $4 \%$ молибдена, т. к. известно, что введение вольфрама и/или молибдена способствует увеличению параметров кристаллической решетки сплавов системы XK [8]. 


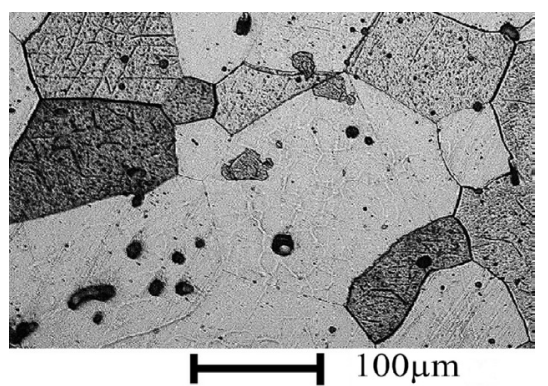

Рис. 1. Структура сплава 22,5X15КС4M после закалки от $1250^{\circ} \mathrm{C}$ и последующего старения при $530^{\circ} \mathrm{C}$.

Fig. 1. The structure of $\mathrm{Fe}-22,5 \% \mathrm{Cr}-15 \% \mathrm{Co}-4 \% \mathrm{Mo}$ alloy after the quenching at $1250^{\circ} \mathrm{C}$ and ageing at $530^{\circ} \mathrm{C}$.

Табл. 1. Магнитные свойства сплавов 22,5X15КС и 22,5X15КСМ в слабых магнитных полях.

Table 1. The magnetic properties of $\mathrm{Fe}-22,5 \% \mathrm{Cr}-15 \% \mathrm{Co}$ and $\mathrm{Fe}-22,5 \% \mathrm{Cr}-15 \% \mathrm{Co}-4 \% \mathrm{Mo}$ alloys in weak fields.

\begin{tabular}{|c|c|c|c|c|c|}
\hline \multirow{3}{*}{$\begin{array}{l}\text { Сплав } \\
\text { Alloy }\end{array}$} & \multirow{3}{*}{ 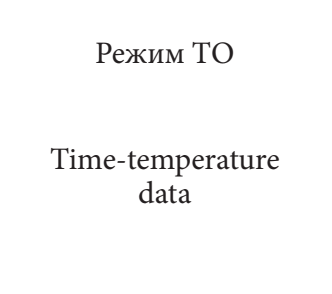 } & \multicolumn{4}{|c|}{$\begin{array}{c}\text { Свойства по методике } \\
H_{m}=-90 \ldots+90 \mathrm{~A} / \mathrm{cm} \\
\text { Properties by the procedure } \\
H_{m}=-90 \ldots+90 \mathrm{~A} / \mathrm{cm}\end{array}$} \\
\hline & & $H_{m}$ & $H_{c}$ & $B_{m}$ & $B_{r}$ \\
\hline & & \multicolumn{2}{|c|}{$\begin{array}{l}\mathrm{A} / \mathrm{cm} \\
\mathrm{A} / \mathrm{cm}\end{array}$} & \multicolumn{2}{|c|}{$\begin{array}{l}\text { Тл } \\
\text { Tl }\end{array}$} \\
\hline \multirow{2}{*}{ 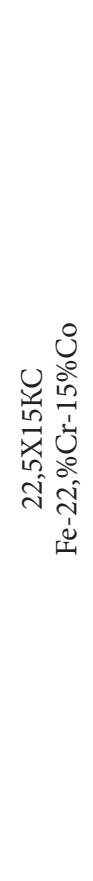 } & $\begin{array}{c}\text { закалка } 1250^{\circ} \mathrm{C}, 20 \text { мин., } \\
\text { в воде, старение } 620^{\circ} \mathrm{C}, \\
1 \text { ч., в воде, } \\
\text { старение } 600^{\circ} \mathrm{C}, 30 \text { мин., } \\
\text { на воздухе } \\
\text { qenching } 1250^{\circ} \mathrm{C}, \\
20 \text { min., water, } \\
\text { ageing } 620^{\circ} \mathrm{C}, 1 \mathrm{~h} ., \\
\text { water, ageing } 600^{\circ} \mathrm{C}, \\
30 \text { min., air }\end{array}$ & 90 & 38 & 1,3 & 0,38 \\
\hline & $\begin{array}{c}\text { закалка } 1250^{\circ} \mathrm{C}, 20 \text { мин., } \\
\text { в воде, старение } 530^{\circ} \mathrm{C}, \\
2 \text { ч., в воде, } \\
\text { старение } 620^{\circ} \mathrm{C}, 1 \text { ч., } \\
\text { в воде, старение } 600^{\circ} \mathrm{C}, \\
30 \text { мин., на воздухе } \\
\text { queching } 1250^{\circ} \mathrm{C}, \\
20 \text { min., water, } \\
\text { ageing } 530^{\circ} \mathrm{C}, 2 \mathrm{~h} ., \\
\text { water, ageing } 620^{\circ} \mathrm{C}, 1 \mathrm{~h} ., \\
\text { water, geing } 600^{\circ} \mathrm{C}, \\
30 \text { min., air }\end{array}$ & 90 & 54 & 0,86 & 0,44 \\
\hline 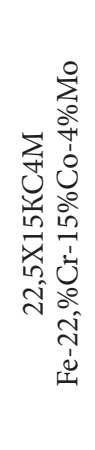 & $\begin{array}{c}\text { закалка } 1250^{\circ} \mathrm{C}, 20 \mathrm{мин.,} \\
\text { в воде, старение } 530^{\circ} \mathrm{C}, \\
2 \text { ч., в воде, } \\
\text { старение } 620^{\circ} \mathrm{C}, 1 \text { ч., } \\
\text { в воде, старение } 600^{\circ} \mathrm{C}, \\
30 \text { мин., на воздухе } \\
\text { queching } 1250^{\circ} \mathrm{C}, \\
20 \text { min., water, } \\
\text { ageing } 530^{\circ} \mathrm{C}, 2 \mathrm{~h} ., \\
\text { water, ageing } 620^{\circ} \mathrm{C}, 1 \mathrm{~h} ., \\
\text { water, ageing } 600^{\circ} \mathrm{C}, \\
30 \text { min., air }\end{array}$ & 90 & 32 & 1,1 & 0,85 \\
\hline
\end{tabular}

Проведение предварительного низкотемпературного отпуска при $530^{\circ} \mathrm{C}$ способствует росту магнитных характеристик. Введение молибдена способствует повышению $H_{c}$ и $B_{r}$. Изменение гистерезисных свойств связано с перераспределением молибдена в твердом растворе. По данным [6, 7], молибден переходит в слабомагнитную фазу. Наличие в структуре сплавов сильномагнитной и слабомагнитной фазы, а так же периодическое расположение частиц фазы с повышенным содержанием Со в фазе с высокой концентрацией Сr обеспечивает достигнутый уровень магнитных свойств.

Без проведения предварительного упорядочения все характеристики гребневых сплавов были в 1,5-2 раза ниже.

Рост коэрцитивной силы начинается при температуре старения $600^{\circ} \mathrm{C}$ и ниже. Происходят изменения размеров и формы выделений фаз $\alpha_{1}$ и $\alpha$, продолжается расслоение. Охлаждение в воде способствует стабилизации структуры: сохраняется высокие значение индукции, но коэрцитивная сила не растёт. При охлаждении на воздухе в диапазоне температур $580-600^{\circ} \mathrm{C}$ происходит рост $H_{c}$, что обусловлено дораспадом, в процессе которого, по-видимому, изменяется форма, размеры и соотношение фаз.

Кривая намагничивания порошкового сплава 25X15КСА представлена в [9]. Отличительной особенностью сплавов 22,5Х15КС4М являются повышенные значения индукции и коэрцитивной силы в слабых магнитных полях. Кривая размагничивания представлена на рисунке 2.

В структуре гребневых сплавов после закалки присутствует только $\alpha$-фаза. Многоступенчатый отпуск сплава приводил к спинодальному распаду. Замедленная рентгеновская съемка в $\mathrm{FeK}_{\alpha}$-излучении позволила выявить раздвоение пика $\alpha$-фазы (рис. 3). По данным [10] молибден при распаде концентрируется в слабомагнитной $\alpha_{2}$ фазе, увеличивая разницу параметров решеток, по сравнению с не содержащим Мо аналогом. После низкотемпературного отпуска в структуре, как и после закалки, выявлена только $\alpha$-фаза. Термическая обработка сплава 22,5Х15КС4М (табл. 1) приводит к повышению разницы объемов сильномагнитной и слабомагнитной фаз. Параметры решетки $\alpha_{1}$ и $\alpha_{2}$ фаз определены из сле-

\section{Residual induction (Br), Tl}

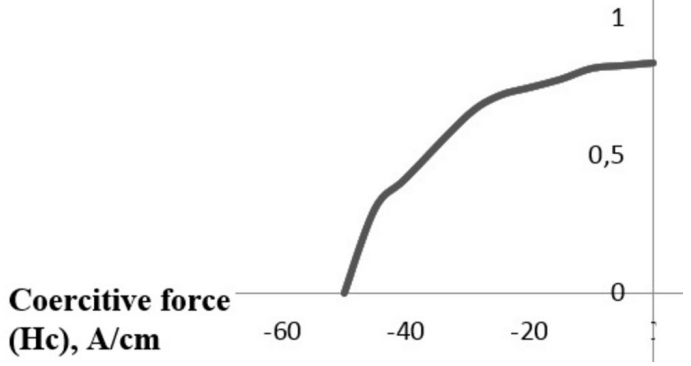

Рис. 2. Кривая размагничивания сплава 22,5Х15КС4М.

Fig. 2. The demagnetization curve of Fe- $22,5 \% \mathrm{Cr}-15 \% \mathrm{Co}-4 \% \mathrm{Mo}$ alloy. 
дующих предположений: $1-$ при образовании $\alpha_{1}$ и $\alpha_{2}$ фаз удельный объем не изменяется; $2-$ у порошковых материалов соотношение между параметрами $a$ и $c$ в тетрагональных фазах $\alpha_{1}$ и $\alpha_{2}$ такое же, как в деформированных сплавах. Указанные предположения позволили определить параметры $a$ и $c$ (таблица 2).

Итак, добавки молибдена способствуют ускорению диффузионных процессов, контролирующих усадку; повышение плотности при спекании молибденсодержащих гребневых сплавов по-видимому обусловлено увеличением области существования $\alpha$-фазы и расширением температурных интервалов образования «исчезающей» жидкой фазы, образующейся за счет контактного плавления; предварительное упорядочение закаленных гребневых сплавов сопровождается фрагментацией зеренной структуры $\alpha$-фазы и обеспечивает после полного цикла термической обработки высокий уровень свойств гребневых сплавов для ГД; экспериментально доказано, что на стадии старения порошковых гребневых сплавов $\alpha$-фаза распадается на две тетрагональные фазы с различными параметрами решетки.

\section{4. Выводы}

1. Предварительный низкотемпературный отпуск в гребневых сплавах системы ХК, сопровождающийся упорядочением и фрагментацией зеренной структуры, оказывает принципиальное влияние на магнитные свойства.

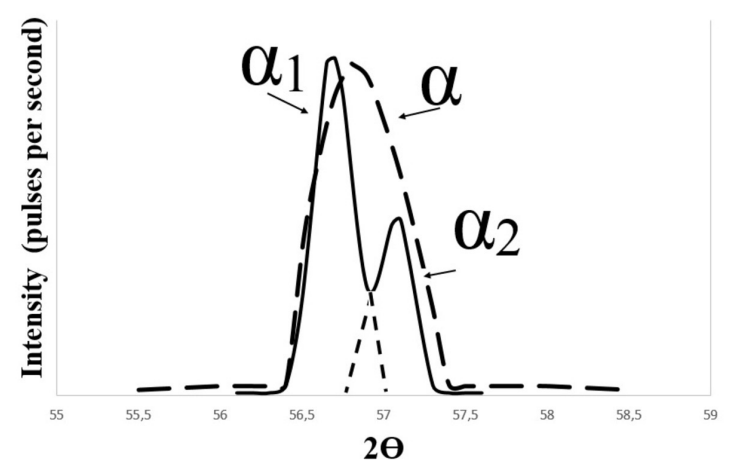

Рис. 3. Рентгенограмма сплавов 22,5Х15КСМ после закалки при $1250^{\circ} \mathrm{C}$ и низкотемпературного отпуска при $530^{\circ} \mathrm{C}$.

Fig. 3. The X-ray diagram of Fe-22,5\%Cr-15\%Co-4\%Mo alloys after quenching at $1250^{\circ} \mathrm{C}$ and low-temperature ageing at $530^{\circ} \mathrm{C}$.

Табл. 2. Параметры решетки для сплава 22,5X15КС4М. Table 2. Lattice parameters for Fe-22,5\%Cr-15\%Co-4\%Mo alloy.

\begin{tabular}{|c|c|}
\hline $\begin{array}{c}\text { «Сильномагнитная» фаза, } \AA \\
\text { «Strong magnetic» phase, } \AA\end{array}$ & $\begin{array}{c}\text { «Слабомагнитная» фаза, } \AA \\
\text { «Weak magnetic» phase, } \AA\end{array}$ \\
\hline $\begin{array}{c}\text { Фаза } \alpha_{1} \quad 2 \Theta=57,06 \\
\text { Phase } \alpha_{1} \quad 2 \Theta=57,06\end{array}$ & $\begin{array}{c}\text { Фаза } \alpha_{2} \quad 2 \Theta=56,65 \\
\text { Phase } \alpha_{2} \quad 2 \Theta=56,65\end{array}$ \\
\hline$a_{1}=2,8678$ & $a_{2}=2,8938$ \\
\hline$c_{1}=2,8534$ & $c_{2}=2,9019$ \\
\hline
\end{tabular}

*индексы «1» и «2» относятся к фазам $\alpha_{1}$ и $\alpha_{2}$ соответственно. *the indices " 1 " and " 2 " refer to the phases $\alpha_{1}$ and $\alpha_{2}$ respectively.
2. Методом рентгеноструктурного анализа порошковых гребневых сплавов доказано образование при расслоении твердого раствора двух наноразмерных сильно и слабомагнитных фаз с собственными параметрами решетки.

3. Разработанные материалы и технологии обеспечивают высокий уровень магнитных и технологических свойств и представляют альтернативу современным деформируемым сплавам систем ХК, ЮНДК, викаллоям и др.

\section{Литература/References}

1. Patent USA № 4601876. C 22C 32/00, 1986.

2. Patent RF №2038918, 09.06.1995. (in Russian) [ПатентРФ № 2038918, 09.06.1995].

3. S. Sugimoto. Improvements of the magnetic properties of equixed Fe-Cr-Co-Mo hard magnets by two-step thermomagnetic treatment // Transactions on magnetics. 1987., Vol. 23.

4. Z. Ahmad. Effect of Mo addition on magnetic properties of Fe - 28Cr - 15Co hard magnets // Physica B 321. 2002. P. $96-103$.

5. Minowa T., Okada M., Homma M. Further studies of the miscibility gap in Fe-Cr-Co permanent magnet system // IEEE Trans. on Magn. 1980 V. 16. N. 3. P. 529-533.

6. Patent RF №2023024, 03.04.1991. (in Russia) [ПатентРФ№ 2023024, 03.04.1991].

7. I. M. Milyaev, V.S. Yusupov, A. I. Milyaev.Eurasian Union of Scientists. Chemistry.,3, (2014), 129-131 (inRussia). [МиляевИ.М., ЮсуповВ.С., МиляевА.И. О концепции самоорганизации высококоорцитивного состояния в магнитотвердых сплавах AlNiCo и $\mathrm{FeCrCo} / /$ Евразийский союз ученых. Химические науки., 2014., № 4., C. $129-131]$.

8. N.A. Belykov, B.E. Vintaikin. VestnikMSTU, (2012), 65-74. (in Russia). [БеляковН.A., ВинтайкинБ.Е. Исследование влияния энергии упругих деформация когерентно сопряженных фаз на фазовое равновесие в сплавах на основе системы $\mathrm{Fe}-\mathrm{Cr}$ Со методами термодинамического моделирования // Вестник МГТУ им. Н. Э. Баумана. Сер. «Естественные науки»., 2012. С. 65 - 74].

9. I. V. Ryaposov. Formirovanie ultramelkokristallicheskoy strukturytermicheskimvozdeistviemnanizkouglerodistye martensitnye staly I magnitnie materialy systemi zhelezochrom-cobalt: Avtoreferat dissertazii na soiskanie stepeni kandidata tehnicheskih nauk. Niznii Novgorod. (2010), 19. (in Russia). [Ряпосов И.В. Формирование ультрамелкокристаллической структуры термическим воздействием на низкоуглеродистые мартенситные стали и магнитные материалы системы железо-хромкобальт: 2010 г.: Автореф. дис.... канд. техн. наук. Нижний Новгород., 2010.-19c].

10. I.S. Belezkaya. Technicalchemistry., (1982), (266), (2). (in Russia). [И.С. Белецкая. Об образовании тетрагональных фаз в магнитожестких сплавах на основе $\mathrm{Fe}$ Cr-Со // Техническая физика. 1982. Т 266., № 2]. 